\title{
Personality and Response to the Financial Crisis
}

\author{
Angela Lee Duckworth \\ University of Pennsylvania \\ and \\ David R. Weir \\ University of Michigan
}

Prepared for the $13^{\text {th }}$ Annual Joint Conference of the Retirement Research Consortium
"Innovations in Retirement Security"
August 4-5, 2011
Washington, DC

This research was supported by a grant from the U.S. Social Security Administration (SSA) as part of the Retirement Research Consortium. The findings and conclusions expressed are solely those of the authors and do not represent the views of the SSA, any agency of the federal government, the University of Pennsylvania, or the University of Michigan. The Health and Retirement Study (HRS) is sponsored by the National Institute on Aging (U01AG009740) and is conducted by the University of Michigan. 


\section{Introduction}

Personality traits, defined as patterns of thinking, feeling, and behaving which are relatively stable across time and situations, have recently been recognized as important predictors of economic outcomes (Borghans, Duckworth, Heckman, \& ter Weel, 2008; Paunonen, 2003). The Big Five taxonomy of personality traits is now widely accepted as the organizational structure of personality traits and distinguishes among traits in the conscientiousness, extraversion, agreeableness, emotional stability, and openness to experience families. This taxonomy has been replicated across cultures (John \& Srivastava, 1999) and developmental stages of the life course (Soto, John, Gosling, \& Potter, 2008).

The personality psychology literature has identified conscientiousness as the Big Five factor most robustly related to academic achievement (Poropat, 2009), job performance (Roberts, Kuncel, Shiner, Caspi, \& Goldberg, 2007), marital stability (Roberts et al., 2007), physical health (Hampson \& Friedman, in press; Hampson, Goldberg, Vogt, \& Dubanoski, 2006), and longevity (Martin, Friedman, \& Schwartz, 2007).

Consistent with these findings, in our previous MRRC project we found Big Five conscientiousness to be more strongly associated with both lifetime earnings and wealth conditional upon earnings, than any other Big Five factor. These associations remained significant even when controlling for years of education, demographics, and measures of cognitive ability. We have since confirmed these findings using structural equation modeling (SEM) to correct estimates for measurement error.

A notable limitation of our prior analyses was the timing of personality assessment (i.e., near or after the end of working life in the HRS). Although personality traits are highly stable in adulthood (7-year test-retest stability about $r=.7$ by the fifth decade of life; Roberts), stronger causal inferences would have been possible had personality traits been measured prior to the outcomes of earnings and retirement savings/investment. One potential pathway for conscientiousness to determine wealth accumulation is adherence to established budgets and saving plans in the face of immediate temptation. The recent financial crisis presented a unique challenge to long-term goals: People who liquidated assets in the trough stood to lose significant 
shares of wealth. In the current project, we attempted to use data from an Internet survey of HRS respondents in the second quarter of 2009 to test how conscientiousness and other Big Five factors prospectively predicted responses to the financial crisis of 2008/09.

A second limitation of our prior analyses was the content of personality assessment in the HRS. The Big Five factors are broad families of personality traits, with component facets of varying relevance to particular outcomes. In the leave-behind psychosocial surveys in 2006 and 2008, only five adjectives (i.e., organized, responsible, hardworking, careless, and thorough) were used to capture Big Five conscientiousness. The facets of perseverance and self-control were not explicitly included. One motivation for investigating with higher-resolution measures these more narrowly specified facets is that they may demonstrate incremental predictive validity for relevant outcomes (Paunonen \& Ashton, 2001). More importantly, understanding which specific traits in the family of Big Five conscientiousness determine economic outcomes, and which do not, can improve the targeting and design of behavioral interventions for "at-risk" individuals.

We (Duckworth) designed an experimental module for the 2010 wave of HRS which includes four items assessing perseverance (sometimes referred to as "grit") and three items assessing self-control. In addition, items were included to assess domain-specific aspects of impulsivity (the obverse of self-control) of theoretical relevance to health and economic outcomes. Specifically, a total of 16 items assess impulsivity in the domains of exercise, food, finances, and interpersonal relations.

Finally, our prior analyses did not relate personality to consumption behavior. Conscientious adults are wealthier even when controlling for lifetime earnings, but as yet research has not confirmed whether conscientious individuals save more and spend less of their income. We therefore merged personality data with data from the Consumption and Activities Mail Survey (CAMS), which was mailed to 5,000 HRS households selected at random from those that participated in HRS 2000. Follow-up questionnaires to the same households were mailed in odd years and refer to consumption and activities the year prior. We computed average self-reported wealth, income, and consumption from all available data. 


\section{Hypotheses}

When controlling for the possible confounds of educational attainment, cognitive ability, and demographic factors --

1. Big Five conscientiousness measured in 2006 and 2008 predicts adaptive decisionmaking (i.e., decisions which preserved wealth) during the 2008/09 financial crisis.

2. Big Five conscientiousness is (inversely) associated with proportion of income spent rather than saved.

3. Perseverance and self-control measured in 2010 demonstrate convergent validity with the Big Five factor of conscientiousness (measured in 2006 and 2008).

4. Perseverance and self-control each demonstrate incremental (inverse) associations, over and beyond Big Five conscientiousness, with proportion of income spent rather than saved.

5. Impulsive behavior in the domain of finances, but not in the domains of exercise, food, and interpersonal relations, is associated with proportion of income spent rather than saved.

\section{Data and Methods}

Our sample derives from the 2006 and 2008 waves of HRS. To be included, a respondent had to complete the self-administered questionnaire with personality measures in either 2006 or 2008, and to be included in the linked Social Security administrative records.

Big Five personality factors were measured using a 26-item questionnaire developed for the Midlife Development Inventory (Lachman \& Bertrand, 2001). HRS participants used a 4-point rating scale to endorse 26 adjectives corresponding to Big Five personality traits of conscientiousness, emotional stability, agreeableness, extraversion, and openness to experience. A total of 14,500 respondents completed the questionnaires.

In September 2001, CAMS wave 1 was mailed to 5,000 households selected at random from households that participated in HRS 2000. In couples households, it was sent to one of the two spouses at random. In September 2003 and October 2005, 2007 and 2009, CAMS waves 2-5 
were sent to the same households. CAMS asked respondents about their spending in each of 32 categories. This represents almost the totality of spending. The rates of item nonresponse were small, and some values could be imputed to zero with considerable confidence, due to the information in the linked HRS data. The resulting spending levels are close to totals from the Consumer Expenditure Survey (CEX) for the age groups 55-74.

Facet-level measures of perseverance and self-control derive from a 2010 experimental module. For each scale, a subset of items was selected from previously validated questionnaires for perseverance (Duckworth, Peterson, Matthews, \& Kelly, 2007) and self-control (Tangney, Baumeister, \& Boone, 2004). Similarly, items assessing four types of domain-specific impulsivity behavior were selected from a previously validated questionnaire (Tsukayama, Duckworth \& Kim, 2011). As of this report, data are available for $N=1587$ cases, though the majority of these were new participants for whom CEX and prior Big Five personality data were not available.

In all regression analyses we controlled for birth year, sex, ethnicity, HRS entry cohort, years of education, and a composite measure of cognitive ability encompassing four cognitive measures that were standardized and averaged: episodic memory (sum of immediate and delayed word recall), mental status (backward counting task), numeracy, and vocabulary. We took the first observation in the panel on each of these cognitive measures to minimize the impact of agerelated decline.

\section{Results}

Hypothesis 1. Insufficient data. For analyses where data were available from most Internet respondents, the sample size was about $N=750$. However, for many other questions, the sample size was considerably smaller because of missing data on the Internet survey (e.g., retirement questions inapplicable to many respondents). There were very few associations with any personality traits that were both significant and theoretically interpretable, and none of these associations survived correction for multiple comparisons. 
Hypothesis 2. Yes. Controlling for race, gender, birthdate, HRS cohort, and years of education, (log) wealth, and cognitive ability, the ratio of (log) average consumption to (log) average income was associated inversely with Big Five conscientiousness $(\beta=-.06)$ and positively with Big Five openness to experience $(\beta=.08)$. The pattern of findings was similar without controlling for wealth and whether or not outliers are removed. In other words, more conscientious (e.g., hardworking, dependable) adults spend less of their income, whereas individuals who are "sophisticated" and "adventurous” spend more.

Hypothesis 3. Yes. Among $N=272$ participants on whom data for both the experimental module and personality were available, conscientiousness was correlated with both perseverance ( $r=$ $.31)$ and self-control $(r=.30)$. However, while the coefficient alpha for the 5 -item grit scale was adequate .67, the coefficient alpha for the 3-item self-control scale was only .37, suggesting poor reliability.

Hypothesis 4. Insufficient data. Perseverance was inversely associated with the ratio of (log) average consumption to (log) average income $(\beta=-.14)$ as well as log income $(\beta=.17)$, but only the latter association reached significance $(\beta=.12)$ when controlling for Big Five personality among the $N=182$ participants for whom Big Five personality data are also available. Selfcontrol was not significantly associated with income, wealth, or the ratio of consumption to income, though high measurement error likely attenuated these associations.

Hypothesis 5. Yes. Impulsivity in the domain of finances was associated with the ratio of (log) average consumption to (log) average income $(\beta=.15)$ when controlling for race, gender, birthdate, HRS cohort, and years of education, (log) wealth, cognitive ability, and conscientiousness (which is no longer a significant predictor once impulsivity in finance is controlled). All domain-specific impulsivity measures demonstrated convergent validity with domain-general self-control (rs from -.24 to -.50) and with conscientiousness ( $r$ s from -.17 to .24).

\section{Discussion}


Our findings support the hypothesis that personality influences financial outcomes among older adults. Conscientious individuals, who earn more money and end up wealthier than other individuals, save more (and, hence, spend less) of their income. Furthermore, the effect of conscientiousness on consumption can be at least partly explained by self-controlled behavior in the domain of finances. That is, more conscientious individuals less frequently "buy things on impulse," "spend too much money," "buy things I hadn’t planned to buy," and "buy things I don't really need." These behavioral tendencies in turn predict the proportion of earnings spent vs. saved.

Future research is needed to test whether conscientious adults, in addition to saving more, invest more wisely. Our attempt to pursue this question using data from a very small subsample of HRS participants who responded to an Internet survey following the financial crisis was unsuccessful. Likewise, additional research is needed to illuminate the relative importance of distinct facets of Big Five conscientiousness to economic outcomes.

In general, deeper insight into specific aspects of personality that determine economic behavior should sharpen policy and intervention efforts aimed at improving the financial security and well-being of older adults. 


\section{References}

Borghans, L., Duckworth, A. L., Heckman, J. J., \& ter Weel, B. (2008). The economics and psychology of personality traits. Journal of Human Resources, 43, 972-1059.

Duckworth, A. L., Peterson, C., Matthews, M. D., \& Kelly, D. R. (2007). Grit: Perseverance and passion for long-term goals. Journal of Personality and Social Psychology, 92, 10871101.

Hampson, S. E., \& Friedman, H. S. (in press). Personality and health: A life span perspective. In O. P. John, R. W. Robins, \& L. Pervin (Eds.), The handbook of personality (3rd ed.). New York, NY: Guilford.

Hampson, S. E., Goldberg, L. R., Vogt, T. M., \& Dubanoski, J. P. (2006). Forty years on: Teachers' assessments of children's personality traits predict self-reported health behaviors and outcomes at midlife. Health Psychology, 25, 57-64.

John, O. P., \& Srivastava, S. (1999). The Big Five Trait taxonomy: History, measurement, and theoretical perspectives. In L. A. Pervin \& O. P. John (Eds.), Handbook of personality: Theory and research (2nd ed., pp. 102-138). New York, NY, US: Guilford Press.

Lachman, M. E. \& Bertrand, R. M. (2001). Personality and the self in midlife. In M. E. Lackman (Ed.) Handbook of midlife development. New York, NY: John Wiley \& Sons.

Martin, L. R., Friedman, H. S., \& Schwartz, J. E. (2007). Personality and mortality risk across the life span: The importance of conscientiousness as a biopsychosocial attribute. Health Psychology, 26, 428-436.

Paunonen, S. V. (2003). Big Five factors of personality and replicated predictions of behavior. Journal of Personality and Social Psychology, 84, 411-422.

Paunonen, S. V., \& Ashton, M. C. (2001). Big Five factors and facets and the prediction of behavior. Journal of Personality and Social Psychology, 81, 524-539.

Poropat, A. E. (2009). A meta-analysis of the five-factor model of personality and academic performance. Psychological Bulletin, 135, 322-338.

Roberts, B. W., Kuncel, N. R., Shiner, R., Caspi, A., \& Goldberg, L. R. (2007). The power of personality: The comparative validity of personality traits, socioeconomic status, and cognitive ability for predicting important life outcomes. Perspectives on Psychological Science, 2, 313-345.

Soto, C. J., John, O. P., Gosling, S. D., \& Potter, J. (2008). The developmental psychometrics of Big Five self-reports: Acquiescence, factor structure, coherence, and differentiation from ages 10 to 20. Journal of Personality and Social Psychology, 94, 718-737.

Tangney, J. P., Baumeister, R. F., \& Boone, A. L. (2004). High self-control predicts good adjustment, less pathology, better grades, and interpersonal success. Journal of Personality, 72, 271-322.

Tsukayama, E., Duckworth, A. L., \& Kim, B. E. (in press). Resisting everything but temptation: Domain-specific and domain-general aspects of self-control. European Journal of Personality. 


\section{Appendix}

$\underline{\text { Calculation of ratio of consumption to income from CAMS data and associated histograms }}$

COMPUTE log_wlth_avg=lg10(mean(wlth2000,wlth2002,wlth2004,wlth2006,wlth2008)). EXECUTE.

COMPUTE log_inc_avg=lg10(mean(inc1999,inc2001,inc2003,inc2005,inc2007)).

EXECUTE.

COMPUTE log_cex_avg=lg10(mean(cex2001,cex2003,cex2005,cex2007,cex2009)).

EXECUTE.

COMPUTE ratio_log_cex_log_inc = log_cex_avg/log_inc_avg.

EXECUTE.

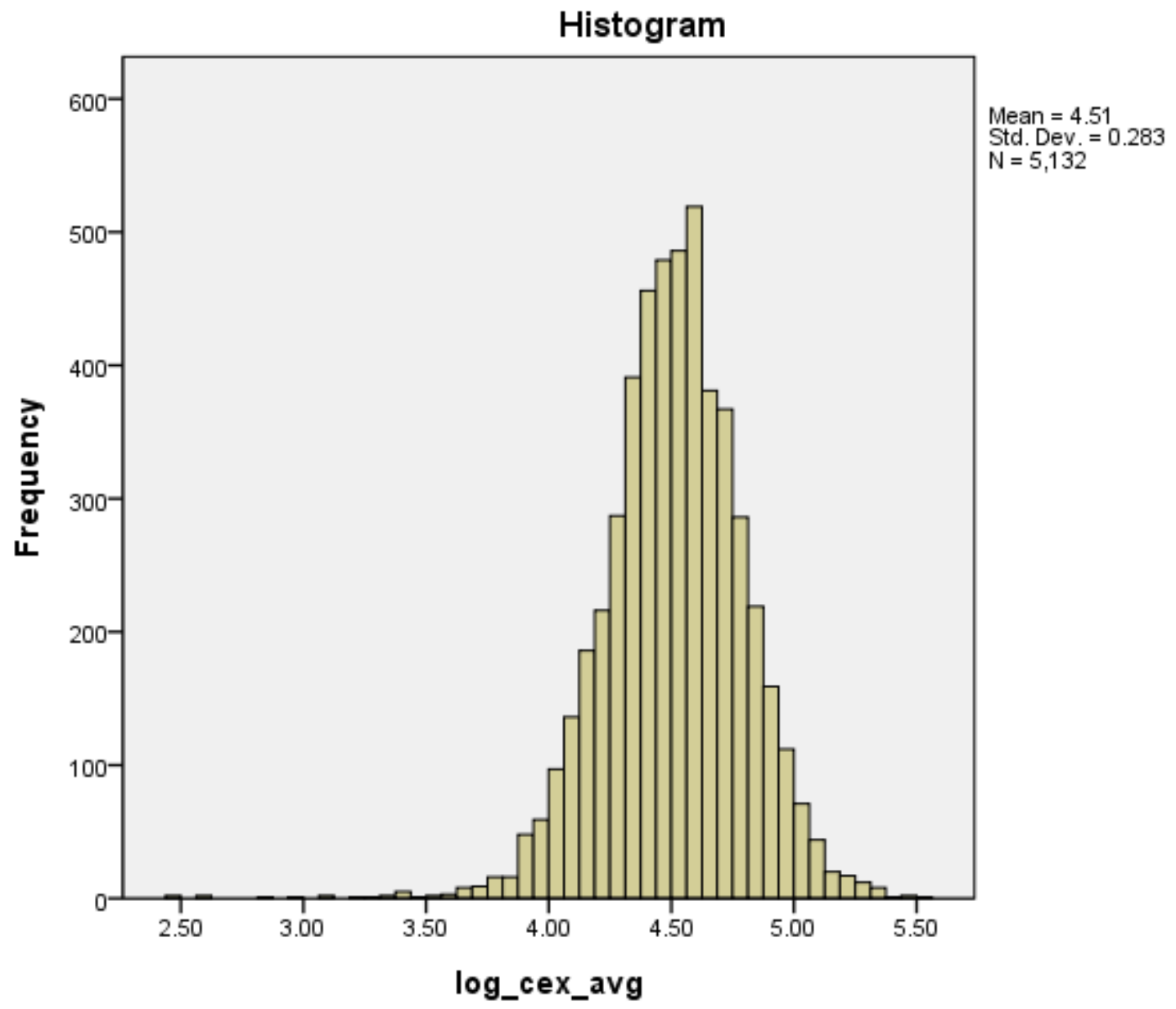


Histogram

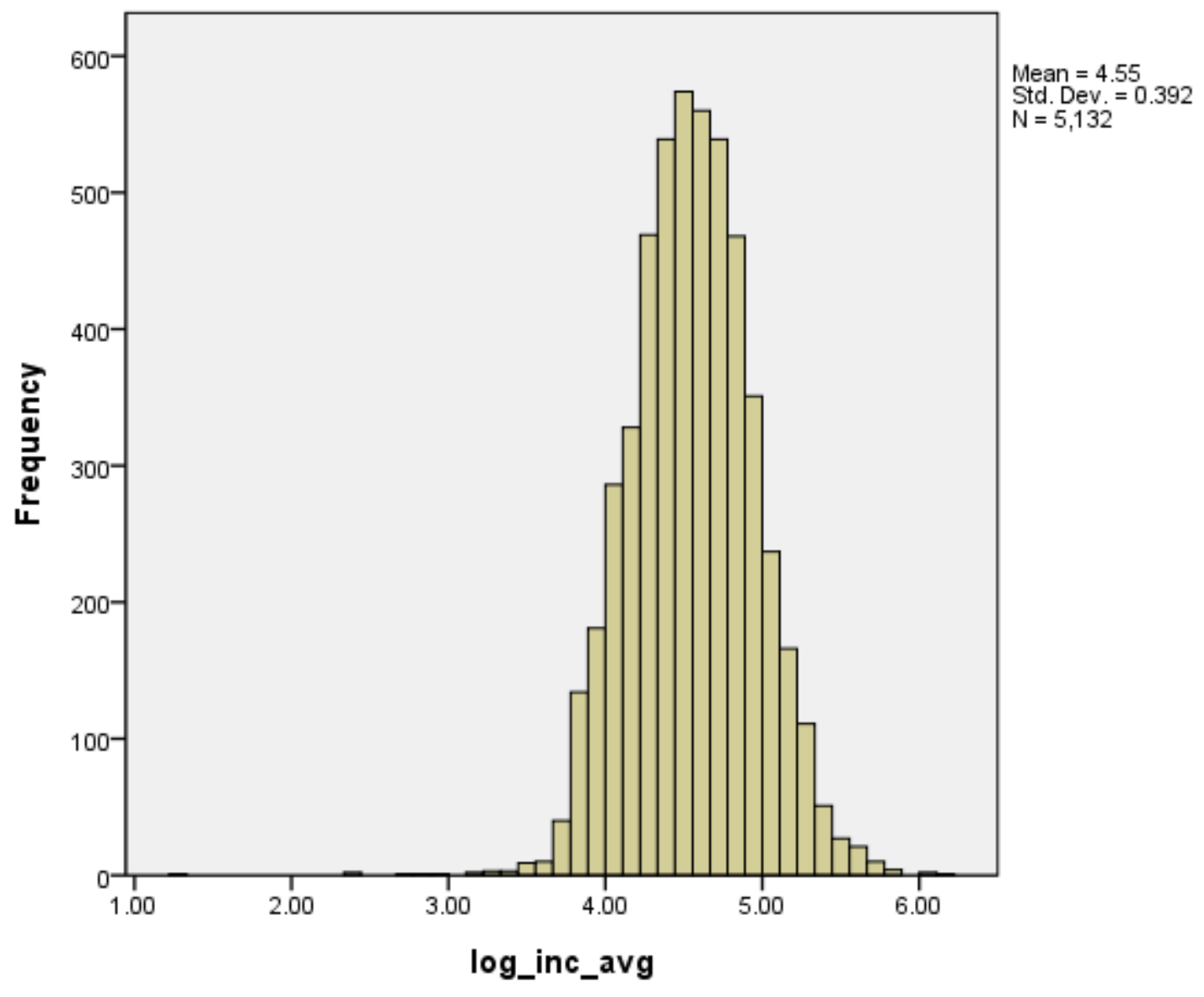


Histogram

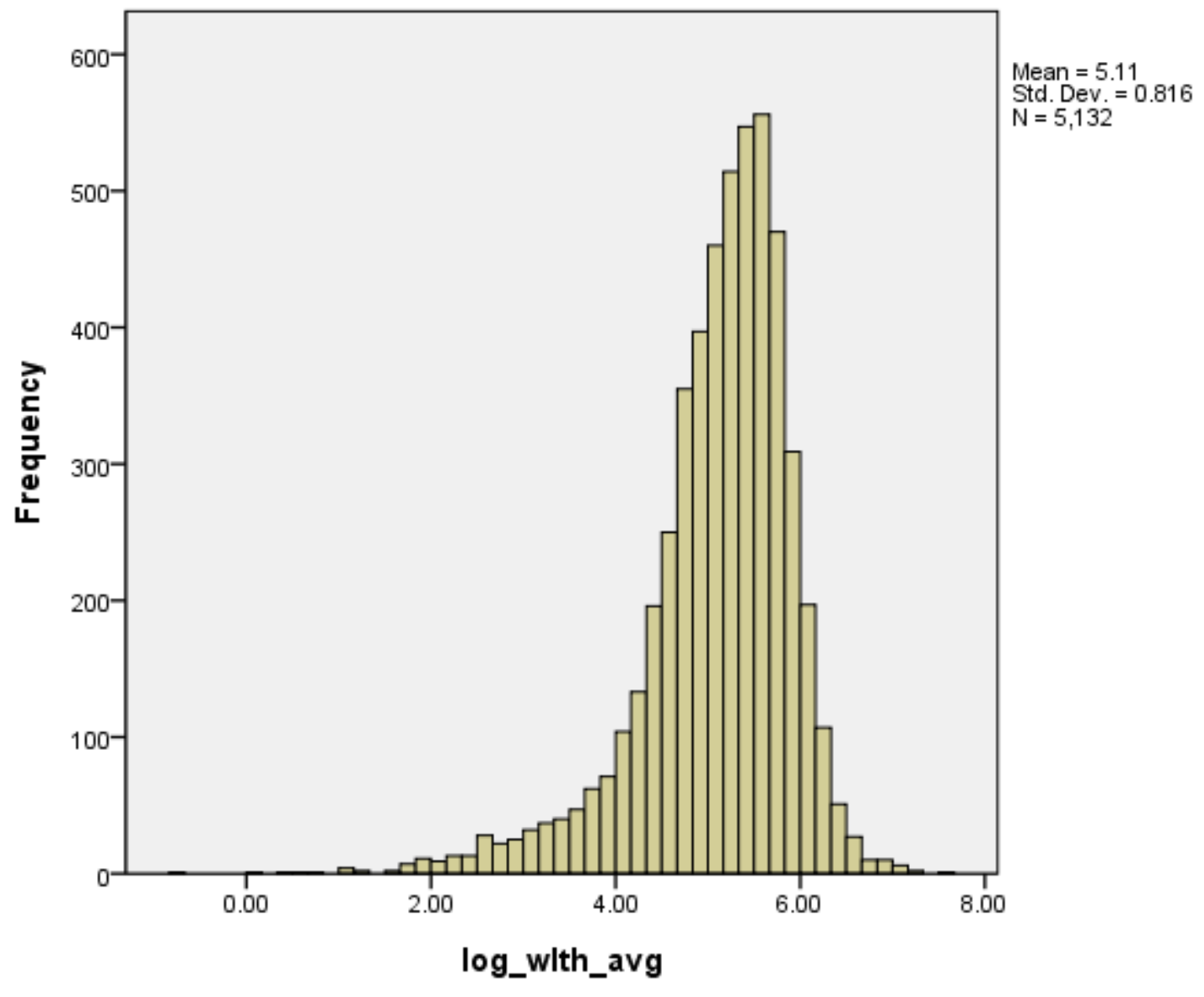


Histogram

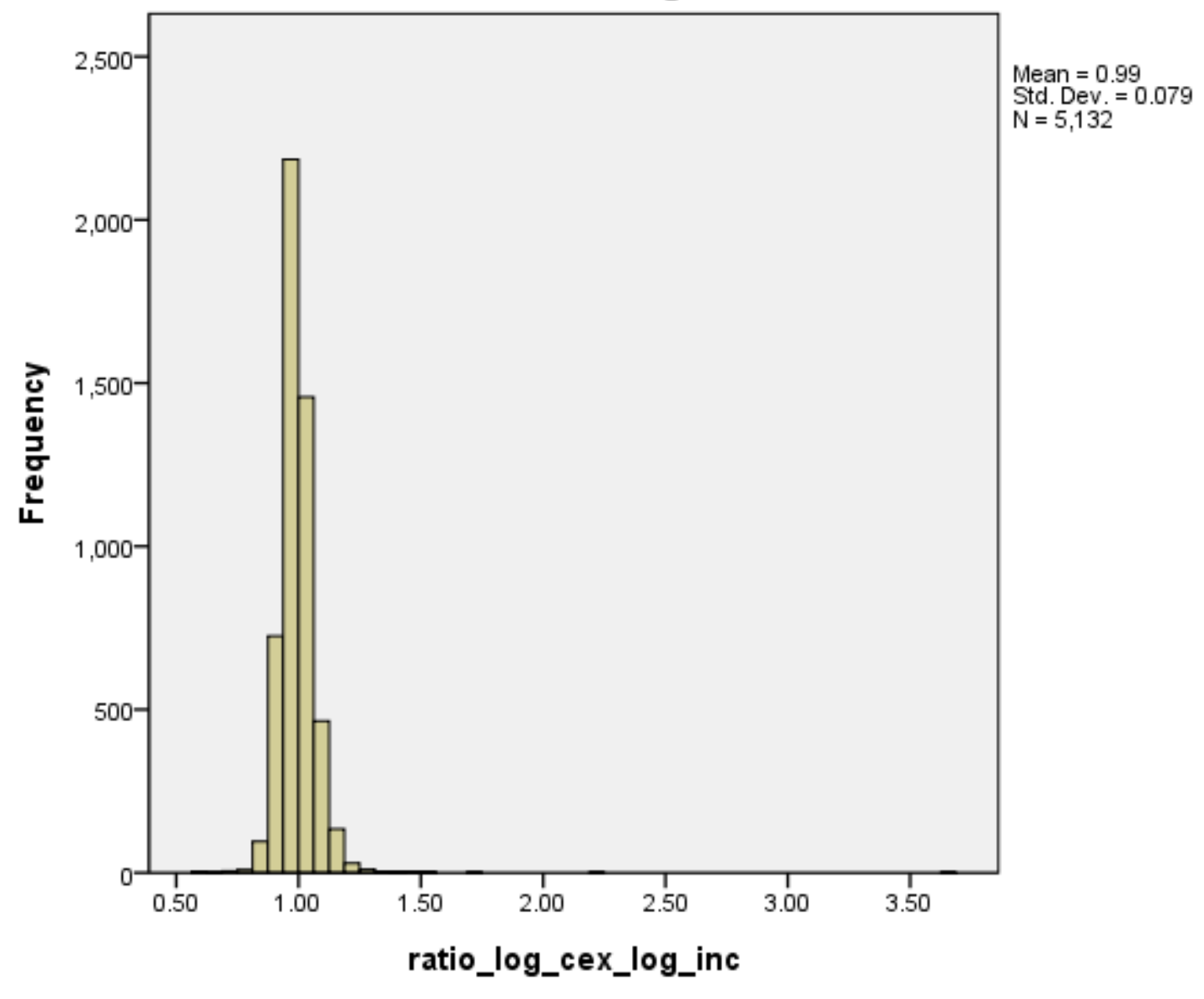


Regression model predicting consumption as a proportion of income from Big Five personality, cognitive ability, and demographic covariates

$\mathrm{N}=2,327$ when using listwise deletion on all variables

$F(16,2310)=38.43, \mathrm{p}<.001$

Coefficients $^{\mathrm{a}}$

\begin{tabular}{|c|c|c|c|c|c|c|}
\hline \multirow{2}{*}{\multicolumn{2}{|c|}{ Model }} & \multicolumn{2}{|c|}{$\begin{array}{l}\text { Unstandardized } \\
\text { Coefficients }\end{array}$} & \multirow{2}{*}{ 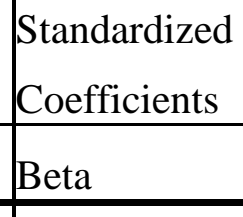 } & \multirow[b]{2}{*}{ t } & \multirow[b]{2}{*}{ Sig. } \\
\hline & & B & Std. Error & & & \\
\hline \multirow[t]{17}{*}{1} & (Constant) & 3.060 & .376 & & 8.140 & .000 \\
\hline & birthdate: year & .001 & .000 & -.188 & -5.249 & .000 \\
\hline & gender & .013 & .002 & .124 & 6.075 & .000 \\
\hline & hisp & .011 & .004 & .051 & 2.562 & .010 \\
\hline & black & .014 & .003 & .085 & 4.220 & .000 \\
\hline & edyrs & -.003 & .000 & -.139 & -5.727 & .000 \\
\hline & cohort $==2$ & .001 & .005 & .006 & .260 & .795 \\
\hline & cohort $==3$ & .002 & .005 & .017 & .410 & 682 \\
\hline & cohort $==4$ & -.004 & .006 & -.027 & -.679 & .497 \\
\hline & cohort= $=5$ & -.001 & .007 & -.007 & -.163 & .871 \\
\hline & log_wlth_avg & -.015 & .001 & -.217 & -10.173 & .000 \\
\hline & agreeab & .001 & .003 & .013 & .555 & .579 \\
\hline & extrov & -.001 & .002 & -.015 & -.603 & .547 \\
\hline & neurot & .003 & .002 & .030 & 1.517 & 129 \\
\hline & consci & -.007 & .002 & -.062 & -2.767 & .006 \\
\hline & open & .008 & .002 & .080 & 3.263 & .001 \\
\hline & $\begin{array}{l}\text { Cognitive scores } \\
\text { standardized and } \\
\text { averaged }\end{array}$ & -.003 & .002 & -.038 & -1.640 & .101 \\
\hline
\end{tabular}

a. Dependent Variable: ratio_log_cex_log_inc 
Bivariate correlations among perseverance (grit), self-control, Big Five personality, and cognitive ability

\begin{tabular}{|c|c|c|c|c|c|c|c|c|c|}
\hline & & Grit & \begin{tabular}{|l} 
SelfContr \\
ol
\end{tabular} & agreeab & extrov & neurot & consci & open & $\begin{array}{l}\text { Cognitive } \\
\text { scores } \\
\text { standardize } \\
\text { d and } \\
\text { averaged } \\
\end{array}$ \\
\hline \multirow[t]{3}{*}{ Grit } & $\begin{array}{l}\text { Pearson } \\
\text { Correlation }\end{array}$ & 1 & $.372^{* *}$ & .055 & $.156^{* * 4}$ & $-.323^{* *}$ & $.311^{* *}$ & .110 & .069 \\
\hline & Sig. (2-tailed) & & .000 & 365 & .010 & .000 & .000 & .069 & .269 \\
\hline & $\mathrm{N}$ & 1577 & 1575 & 273 & 273 & 271 & 273 & 273 & 258 \\
\hline \multirow[t]{3}{*}{ SelfControl } & $\begin{array}{l}\text { Pearson } \\
\text { Correlation }\end{array}$ & $.372^{* *}$ & 1 & .103 & .103 & $-.316^{* *}$ & $.299^{* *}$ & $.159^{* *}$ & $.158^{*}$ \\
\hline & Sig. (2-tailed) & .000 & & .091 & .089 & .000 & .000 & .009 & .011 \\
\hline & $\mathrm{N}$ & 1575 & 1575 & 272 & 272 & 270 & 272 & 272 & 257 \\
\hline \multirow[t]{3}{*}{ agreeab } & $\begin{array}{l}\text { Pearson } \\
\text { Correlation }\end{array}$ & .055 & 103 & 1 & $.566^{* *}$ & $-.113^{* *}$ & $.435^{* *}$ & $.417^{* *}$ & .028 \\
\hline & Sig. (2-tailed) & .365 & .091 & & .000 & .000 & .000 & .000 & .126 \\
\hline & $\mathrm{N}$ & 273 & 272 & 3768 & 3766 & 3745 & 3761 & 3756 & 3088 \\
\hline \multirow[t]{3}{*}{ extrov } & $\begin{array}{l}\text { Pearson } \\
\text { Correlation }\end{array}$ & $.156^{* *}$ & .103 & $.566^{* *}$ & 1 & $-.217^{* *}$ & $.382^{* *}$ & $.540^{* *}$ & .015 \\
\hline & Sig. (2-tailed) & .010 & .089 & .000 & & .000 & .000 & .000 & .395 \\
\hline & $\mathrm{N}$ & 273 & 272 & 3766 & 3769 & 3745 & 3760 & 3756 & 3088 \\
\hline \multirow[t]{3}{*}{ neurot } & $\begin{array}{l}\text { Pearson } \\
\text { Correlation }\end{array}$ & & $-.316^{* *}$ & $-.113^{* *}$ & $-.217^{* *}$ & 1 & $-.253^{* * *}$ & $-.187^{* *}$ & $-.117^{* *}$ \\
\hline & Sig. (2-tailed) & .000 & .000 & .000 & .000 & & .000 & .000 & .000 \\
\hline & $\mathrm{N}$ & 271 & 270 & 3745 & 3745 & 3745 & 3741 & 3738 & 3068 \\
\hline \multirow[t]{3}{*}{ consci } & $\begin{array}{l}\text { Pearson } \\
\text { Correlation }\end{array}$ & & $.299^{* *}$ & $.435^{* *}$ & $.382^{* *}$ & $-.253^{* *}$ & 1 & $.453^{* * *}$ & $.175^{* *}$ \\
\hline & Sig. (2-tailed) & .000 & .000 & .000 & .000 & .000 & & .000 & .000 \\
\hline & $\mathrm{N}$ & 273 & 272 & 3761 & 3760 & 3741 & 3761 & 3752 & 3082 \\
\hline \multirow[t]{3}{*}{ open } & $\begin{array}{l}\text { Pearson } \\
\text { Correlation }\end{array}$ & .110 & $.159^{* *}$ & $.417^{* *}$ & $.540^{* *}$ & $-.187^{* *}$ & $.453^{* *}$ & 1 & \\
\hline & Sig. (2-tailed) & .069 & .009 & .000 & .000 & .000 & .000 & & .000 \\
\hline & $\mathrm{N}$ & 273 & 272 & 3756 & 3756 & 3738 & 3752 & 3757 & 3080 \\
\hline \multirow{3}{*}{$\begin{array}{l}\text { Cognitive scores } \\
\text { standardized and } \\
\text { averaged }\end{array}$} & $\begin{array}{l}\text { Pearson } \\
\text { Correlation }\end{array}$ & .069 & $158^{*}$ & .028 & .015 & $-.117^{* *}$ & $.175^{* *}$ & $.191^{* *}$ & 1 \\
\hline & Sig. (2-tailed) & .269 & .011 & .126 & 395 & .000 & .000 & .000 & \\
\hline & $\mathrm{N}$ & 258 & 257 & 3088 & 3088 & 3068 & 3082 & 3080 & 3427 \\
\hline
\end{tabular}


Internal reliability of grit and self-control scales given in 2010 experimental module

Reliability Statistics

\begin{tabular}{|r|r|}
\hline Cronbach's Alpha & N of Items \\
\hline .674 & 5 \\
\hline
\end{tabular}

Item-Total Statistics

\begin{tabular}{|l|r|r|r|r|}
\hline & $\begin{array}{c}\text { Scale Mean if Item } \\
\text { Deleted }\end{array}$ & $\begin{array}{c}\text { Scale Variance if } \\
\text { Item Deleted }\end{array}$ & $\begin{array}{c}\text { Corrected Item-Total } \\
\text { Correlation }\end{array}$ & $\begin{array}{c}\text { Cronbach's Alpha if } \\
\text { Item Deleted }\end{array}$ \\
\hline new ideas and projects distract & 14.7703 & 10.754 & .415 & .628 \\
obsessed but lose interest & 14.4244 & 9.703 & .563 & .561 \\
set goal but pursue different one & 14.3945 & 10.352 & .472 & .602 \\
maintain focus on long projects & 14.4796 & 8.808 & .603 \\
persistent & 14.2297 & 12.525 & .533 \\
\hline
\end{tabular}

Reliability Statistics

\begin{tabular}{|r|r|}
\hline Cronbach's Alpha & N of Items \\
\hline 367 & 3 \\
\hline
\end{tabular}

Item-Total Statistics

\begin{tabular}{|l|r|r|r|r|}
\hline & $\begin{array}{c}\text { Scale Mean if Item } \\
\text { Deleted }\end{array}$ & $\begin{array}{c}\text { Scale Variance if } \\
\text { Item Deleted }\end{array}$ & $\begin{array}{c}\text { Corrected Item-Total } \\
\text { Correlation }\end{array}$ & $\begin{array}{c}\text { Cronbach's Alpha if } \\
\text { Item Deleted }\end{array}$ \\
\hline wish for more self-discipline & 7.5723 & 3.281 & .223 & .260 \\
good at resisting temptation & 7.0868 & 4.007 & .131 & .434 \\
cant stop from doing wrong & 6.5897 & 3.673 & .299 & .126 \\
\hline
\end{tabular}


Regression model predicting log income from grit, Big Five personality, cognitive ability, and demographic covariates

$N=182$ when using listwise deletion on all variables

$F(17,164)=15.44, p<.001$

\begin{tabular}{|c|c|c|c|c|c|c|}
\hline \multicolumn{7}{|c|}{ Coefficients $^{a}$} \\
\hline \multirow[b]{2}{*}{ Mode } & & \multicolumn{2}{|c|}{ Unstandardized Coefficients } & \multirow{2}{*}{$\begin{array}{c}\text { Standardized } \\
\text { Coefficients } \\
\text { Beta } \\
\end{array}$} & \multirow[b]{2}{*}{$\mathrm{t}$} & \multirow[b]{2}{*}{ Sig. } \\
\hline & & $\mathrm{B}$ & Std. Error & & & \\
\hline \multirow[t]{19}{*}{1} & (Constant) & -4.624 & 7.296 & & -.634 & .527 \\
\hline & gender & -.098 & .041 & -.128 & -2.379 & .019 \\
\hline & birthdate: year & .004 & .004 & .099 & .976 & .331 \\
\hline & hisp & -.023 & .070 & -.017 & -.324 & .746 \\
\hline & black & -.058 & .056 & -.057 & -1.048 & .296 \\
\hline & edyrs & .030 & .008 & .246 & 3.740 & .000 \\
\hline & cohort==2 & .071 & .082 & .066 & .866 & .388 \\
\hline & cohort==3 & .095 & .090 & .134 & 1.054 & .293 \\
\hline & cohort $==4$ & .147 & .122 & .141 & 1.205 & .230 \\
\hline & cohort $==5$ & .198 & .137 & .179 & 1.440 & .152 \\
\hline & log_wlth_avg & .242 & .028 & .508 & 8.688 & .000 \\
\hline & Cognitive scores standardized and & .018 & .033 & .035 & .545 & .586 \\
\hline & averaged & & & & & \\
\hline & Grit & .051 & .024 & .118 & 2.069 & .040 \\
\hline & agreeab & .077 & .059 & .088 & 1.308 & .193 \\
\hline & extrov & -.035 & .048 & -.052 & -.726 & .469 \\
\hline & neurot & -.020 & .031 & -.037 & -.653 & .515 \\
\hline & consci & .065 & .049 & .083 & 1.325 & .187 \\
\hline & open & -.034 & .039 & -.055 & -.877 & .382 \\
\hline
\end{tabular}

a. Dependent Variable: log_inc_avg 
Regression model predicting consumption as a proportion of income from grit, Big Five personality, cognitive ability, and demographic covariates

$N=182$ when using listwise deletion on all variables

$F(17,164)=5.56, p<.001$

\begin{tabular}{|c|c|c|c|c|c|c|}
\hline \multicolumn{7}{|c|}{ Coefficients $^{a}$} \\
\hline \multirow{2}{*}{\multicolumn{2}{|c|}{ Model }} & \multicolumn{2}{|c|}{ Unstandardized Coefficients } & \multirow{2}{*}{$\begin{array}{c}\text { Standardized } \\
\text { Coefficients } \\
\text { Beta } \\
\end{array}$} & \multirow[b]{2}{*}{$\mathrm{t}$} & \multirow[b]{2}{*}{ Sig. } \\
\hline & & B & Std. Error & & & \\
\hline 1 & (Constant) & 3.580 & 1.542 & & 2.322 & .021 \\
\hline & gender & .010 & .009 & .076 & 1.099 & .273 \\
\hline & birthdate: year & -.001 & .001 & -.204 & -1.565 & .119 \\
\hline & hisp & -.006 & .015 & -.025 & -.373 & .710 \\
\hline & black & .038 & .012 & .223 & 3.210 & .002 \\
\hline & edyrs & -.002 & .002 & -.081 & -.964 & .336 \\
\hline & cohort==2 & -.022 & .017 & -.126 & -1.286 & .200 \\
\hline & cohort==3 & -.008 & .019 & -.069 & -.419 & .675 \\
\hline & cohort==4 & -.004 & .026 & -.021 & -.141 & .888 \\
\hline & cohort==5 & .002 & .029 & .010 & .064 & .949 \\
\hline & log_wlth_avg & -.023 & .006 & -.299 & -3.975 & .000 \\
\hline & Cognitive scores standardized and & -.007 & .007 & -.080 & -.966 & .335 \\
\hline & averaged & & & & & \\
\hline & Grit & -.003 & .005 & -.037 & -.508 & .612 \\
\hline & agreeab & -.004 & .012 & -.030 & -.343 & .732 \\
\hline & extrov & -.004 & .010 & -.032 & -.351 & .726 \\
\hline & neurot & .010 & .007 & .115 & 1.567 & .119 \\
\hline & consci & -.015 & .010 & -.118 & -1.465 & .145 \\
\hline & open & .019 & .008 & .186 & 2.333 & .021 \\
\hline
\end{tabular}

a. Dependent Variable: ratio_log_cex_log_inc 
Regression model predicting consumption as a proportion of income from domain-specific impulsive behaviors, Big Five personality, cognitive ability, and demographic covariates

$N=182$ when using listwise deletion on all variables

$F(20,161)=5.66, p<.001$

\begin{tabular}{|c|c|c|c|c|c|c|}
\hline \multicolumn{7}{|c|}{ Coefficients $^{\mathrm{a}}$} \\
\hline \multirow[b]{2}{*}{ Mod } & & \multicolumn{2}{|c|}{ Unstandardized Coefficients } & \multirow{2}{*}{$\begin{array}{c}\text { Standardized } \\
\text { Coefficients } \\
\text { Beta }\end{array}$} & \multirow[b]{2}{*}{$\mathrm{t}$} & \multirow[b]{2}{*}{ Sig. } \\
\hline & & B & Std. Error & & & \\
\hline \multirow[t]{26}{*}{1} & (Constant) & 4.269 & 1.527 & & 2.795 & .006 \\
\hline & Domain-Specific Impulsive Finance & .012 & .006 & .152 & 2.056 & .041 \\
\hline & Behavior & & & & & \\
\hline & Domain-Specific Impulsive Food & .003 & .007 & .034 & .417 & .677 \\
\hline & Behavior & & & & & \\
\hline & Domain-Specific Impulsive Exercise & .009 & .004 & .138 & 1.977 & .050 \\
\hline & Behavior & & & & & \\
\hline & Domain-Specific Impulsive & -.020 & .008 & -.192 & -2.366 & .019 \\
\hline & Interpersonal Behavior & & & & & \\
\hline & gender & .006 & .009 & .045 & .661 & .509 \\
\hline & birthdate: year & -.002 & .001 & -.266 & -2.063 & .041 \\
\hline & hisp & -.004 & .015 & -.017 & -.251 & .802 \\
\hline & black & .038 & .012 & .223 & 3.259 & .001 \\
\hline & edyrs & -.002 & .002 & -.102 & -1.218 & .225 \\
\hline & cohort==2 & -.023 & .017 & -.128 & -1.352 & .178 \\
\hline & cohort==3 & -.004 & .019 & -.030 & -.189 & .851 \\
\hline & cohort $==4$ & .006 & .025 & .032 & .217 & .829 \\
\hline & cohort $==5$ & .007 & .028 & .038 & .246 & .806 \\
\hline & log_wlth_avg & -.021 & .006 & -.262 & -3.509 & .001 \\
\hline & agreeab & -.008 & .012 & -.057 & -.679 & .498 \\
\hline & extrov & -.002 & .010 & -.017 & -.193 & .847 \\
\hline & neurot & .016 & .007 & .184 & 2.446 & .016 \\
\hline & consci & -.011 & .010 & -.088 & -1.153 & .251 \\
\hline & open & .022 & .008 & .210 & 2.668 & .008 \\
\hline & Cognitive scores standardized and & -.004 & .007 & -.052 & -.630 & .530 \\
\hline & averaged & & & & & \\
\hline
\end{tabular}

a. Dependent Variable: ratio_log_cex_log_inc 
Bivariate correlations among domain-specific self-controlled behaviors, conscientiousness, and

\section{self-control}

Correlations

\begin{tabular}{|c|c|c|c|c|c|c|c|}
\hline & & $\begin{array}{c}\text { Domain- } \\
\text { Specific } \\
\text { Impulsive Food } \\
\text { Behavior } \\
\end{array}$ & $\begin{array}{c}\text { Domain-Specific } \\
\text { Impulsive } \\
\text { Finance } \\
\text { Behavior } \\
\end{array}$ & $\begin{array}{c}\text { Domain-Specific } \\
\text { Impulsive } \\
\text { Exercise } \\
\text { Behavior } \\
\end{array}$ & $\begin{array}{c}\text { Domain-Specific } \\
\text { Impulsive } \\
\text { Interpersonal } \\
\text { Behavior } \\
\end{array}$ & consci & SelfControl \\
\hline $\begin{array}{l}\text { Domain-Specific Impulsive } \\
\text { Food Behavior }\end{array}$ & $\begin{array}{l}\text { Pearson Correlation } \\
\text { Sig. (2-tailed) } \\
\mathrm{N}\end{array}$ & $\begin{array}{r}1 \\
1577 \\
\end{array}$ & $\begin{array}{l}.414^{* *} \\
.000 \\
1576 \\
\end{array}$ & $\begin{array}{r}.334^{* *} \\
.000 \\
1574 \\
\end{array}$ & $\begin{array}{l}.380^{* *} \\
.000 \\
1576 \\
\end{array}$ & $\begin{array}{r}-.209^{* *} \\
.001 \\
273 \\
\end{array}$ & $\begin{array}{r}-.497^{* *} \\
.000 \\
1574 \\
\end{array}$ \\
\hline $\begin{array}{l}\text { Domain-Specific Impulsive } \\
\text { Finance Behavior }\end{array}$ & $\begin{array}{l}\text { Pearson Correlation } \\
\text { Sig. (2-tailed) } \\
\mathrm{N}\end{array}$ & $\begin{array}{l}.414^{* *} \\
.000 \\
1576 \\
\end{array}$ & $\begin{array}{r}1 \\
1576 \\
\end{array}$ & $\begin{array}{l}.254^{* *} \\
.000 \\
1574 \\
\end{array}$ & $\begin{array}{l}.353^{* *} \\
.000 \\
1576 \\
\end{array}$ & $\begin{array}{r}-.235^{*} \\
.000 \\
273 \\
\end{array}$ & $\begin{array}{r}-.324^{*} \\
.000 \\
1573 \\
\end{array}$ \\
\hline $\begin{array}{l}\text { Domain-Specific Impulsive } \\
\text { Exercise Behavior }\end{array}$ & $\begin{array}{l}\text { Pearson Correlation } \\
\text { Sig. (2-tailed) } \\
\mathrm{N}\end{array}$ & $\begin{array}{r}.334^{* *} \\
.000 \\
1574 \\
\end{array}$ & $\begin{array}{l}.254^{* *} \\
.000 \\
1574 \\
\end{array}$ & $\begin{array}{r}1 \\
1574 \\
\end{array}$ & $\begin{array}{l}.248^{* *} \\
.000 \\
1574 \\
\end{array}$ & $\begin{array}{r}-.169^{* *} \\
.005 \\
272 \\
\end{array}$ & $\begin{array}{r}-.244^{* *} \\
.000 \\
1571 \\
\end{array}$ \\
\hline $\begin{array}{l}\text { Domain-Specific Impulsive } \\
\text { Interpersonal Behavior }\end{array}$ & $\begin{array}{l}\text { Pearson Correlation } \\
\text { Sig. (2-tailed) } \\
\mathrm{N}\end{array}$ & $\begin{array}{l}.380^{* *} \\
.000 \\
1576 \\
\end{array}$ & $\begin{array}{r}.353^{* *} \\
.000 \\
1576 \\
\end{array}$ & $\begin{array}{r}.248^{* \star} \\
.000 \\
1574 \\
\end{array}$ & 1 & $\begin{array}{r}-.198^{* *} \\
.001 \\
273 \\
\end{array}$ & $\begin{array}{r}-.290^{* *} \\
.000 \\
1573 \\
\end{array}$ \\
\hline consci & $\begin{array}{l}\text { Pearson Correlation } \\
\text { Sig. (2-tailed) } \\
\mathrm{N}\end{array}$ & $\begin{array}{r}-.209^{* *} \\
.001 \\
273 \\
\end{array}$ & $\begin{array}{r}-.235^{* *} \\
.000 \\
273 \\
\end{array}$ & $\begin{array}{r}-.169^{* *} \\
.005 \\
272 \\
\end{array}$ & $\begin{array}{r}-.198^{* *} \\
.001 \\
273 \\
\end{array}$ & $\begin{array}{r}1 \\
3761 \\
\end{array}$ & $\begin{array}{r}.299^{* *} \\
.000 \\
272 \\
\end{array}$ \\
\hline SelfControl & $\begin{array}{l}\text { Pearson Correlation } \\
\text { Sig. (2-tailed) } \\
\mathrm{N}\end{array}$ & $\begin{array}{r}-.497^{* *} \\
.000 \\
1574 \\
\end{array}$ & $\begin{array}{r}-.324^{* *} \\
.000 \\
1573 \\
\end{array}$ & $\begin{array}{r}-.244^{* *} \\
.000 \\
1571\end{array}$ & $\begin{array}{r}-.290^{* *} \\
.000 \\
1573 \\
\end{array}$ & $\begin{array}{l}.299^{* *} \\
.000 \\
272 \\
\end{array}$ & $\begin{array}{r}1 \\
1575 \\
\end{array}$ \\
\hline
\end{tabular}

**. Correlation is significant at the 0.01 level (2-tailed). 